\title{
B-Cell Response during Protozoan Parasite Infections
}

\author{
María C. Amezcua Vesely, Daniela A. Bermejo, Carolina L. Montes, \\ Eva V. Acosta-Rodríguez, and Adriana Gruppi \\ Centro de Investigaciones en Bioquímica Clínica e Inmunología (CIBICI-CONICET), Departamento de Bioquímica Clínica, \\ Facultad de Ciencias Químicas, Universidad Nacional de Córdoba, Haya de la Torre y Medina Allende, Ciudad Universitaria, \\ 5000 Córdoba, Argentina
}

Correspondence should be addressed to Adriana Gruppi, agruppi@fcq.unc.edu.ar

Received 31 August 2011; Accepted 6 October 2011

Academic Editor: Mauricio M. Rodrigues

Copyright (C) 2012 María C. Amezcua Vesely et al. This is an open access article distributed under the Creative Commons Attribution License, which permits unrestricted use, distribution, and reproduction in any medium, provided the original work is properly cited.

\begin{abstract}
In this review, we discuss how protozoan parasites alter immature and mature B cell compartment. B1 and marginal zone (MZ) B cells, considered innate like B cells, are activated during protozoan parasite infections, and they generate short lived plasma cells providing a prompt antibody source. In addition, protozoan infections induce massive B cell response with polyclonal activation that leads to hypergammaglobulnemia with serum antibodies specific for the parasites and self and/or non related antigens. To protect themselves, the parasites have evolved unique ways to evade B cell immune responses inducing apoptosis of $\mathrm{MZ}$ and conventional mature B cells. As a consequence of the parasite induced-apoptosis, the early IgM response and an already establish humoral immunity are affected during the protozoan parasite infection. Moreover, some trypanosomatides trigger bone marrow immature B cell apoptosis, influencing the generation of new mature B cells. Simultaneously with their ability to release antibodies, B cells produce cytokines/quemokines that influence the characteristic of cellular immune response and consequently the progression of parasite infections.
\end{abstract}

\section{B Cells Can Play Protective and Pathogenic Roles in Protozoan Infections}

Host resistance in protozoan infections is dependent on both innate and acquired cell-mediated immune responses. In addition, several studies have implicated B cells and antibodies (Abs) in host survival and protozoan parasite clearance [1-3]. B cells can function as Ab-producing cells but they can also modulate immune responses through critical Abindependent mechanisms that include secretion of cytokines and chemokines as well as antigen presentation [4-6]. Furthermore, B cells can directly modulate dendritic cells and Tcell subsets, and, consequently, they can influence adaptive immunity and the progression of the infection [7]. Accordingly, in protozoan infections B cells may play a protective and a pathological role. In malaria and trypanosome infections, Abs appear to play a famajor role in immunity. In Trypanosoma cruzi and T. brucei gambiense infections, Abdependent cytotoxic reactions against the parasite have been reported [8]. Several studies demonstrated that Abs are responsible for the survival of susceptible animals in the initial phase of T. cruzi infection and for the maintenance of low levels of parasitemia in the chronic phase $[9,10]$. Although Abs were shown to be responsible for clearing the African trypanosomes from the blood of infected animals, recent evidence suggests that the survival time of infected mice does not necessarily correlate with the ability of the animal to produce trypanosome-specific antibody. In general, the parasite-specific immune response mounted during protozoan infections is insufficient to completely eradicate the pathogen, allowing chronic infection.

$\mathrm{B}$ cells do not only play protective roles in protozoan infections. In fact, they are required for the development of Th2 cell response and, consequently, for the susceptibility to infection with Leishmania major [11]. BALB/c uMT mice infected with L. major LV39 mount a Th1 response and present restricted lesion development and contained parasite replication. Adoptive transfer of B cells from BALB/c mice in 
B-cell-deficient BALB/c uMT mice before infection restores susceptibility to L. major LV39 and Th2 cell development in resistant mice.

\section{B-Cell Development}

Given the role for B cells in conditioning the progression of protozoan infections, it is important to understand the kinetics and regulation of the whole B-cell cycle from the development to the differentiation into mature and memory B cells and plasma cells. The humoral immune response has been shown to be two branched providing an innate-like response (involving B1 and marginal zone (MZ) B cells) and an adaptive immune response (involving conventional B2 cells). In the adult, B cells are generated in the bone marrow (BM) and migrate to the periphery at the transitional B-cell stage, when they are still short lived and functionally immature $[12,13]$. Conventional B2-cell development occurs via a series of BM stromal cell-facilitated processes that begin within the hematopoietic stem cell pool and proceed in hierarchical steps of lineage commitment [14]. B lymphopoiesis yields several developmental stages of pre-pro-B, pro-B, pre$B$, and, eventually, immature B cells, which show a high expression of the IgM form of the antigen receptor and low or no expression of the IgD maturation marker. To complete their development, immature B cells migrate through the periphery; however, only $10 \%$ reach the spleen as transitional $\mathrm{B}$ cells of the T1 type [15]. In the spleen, transitional B cells develop into conventional and MZ B cells [16]. B1 cells are efficiently generated in fetal life and during the first few weeks after birth. The fetal liver is an efficient source of B1 cells [17]; however, it is not the only one as a recent study identified a B1-cell precursor in adult BM [18]. Interestingly, protozoan parasite infections can affect the different compartments of B-cell development (summarized in Figure 1), influencing the generation of new mature B cells or their survival and, consequently, the cellular immune response.

\section{Protozoan Parasite Infections Affect BM B-Cell Development}

$\mathrm{BM}$ is the main hematopoietic organ of an adult organism and is able to provide cells of immune systems rapidly in cases of infection. Immature B-cell reduction in BM during an infection would limit the Ab source cells and favour parasite replication and chronicity, so the identification of the mechanisms ruling B-cell depletion represents an important challenge in biomedical research.

We have reported that T. cruzi infection induces a marked loss of immature B cells in the BM and also compromises recently emigrated $B$ cells in the periphery [19]. The depletion of immature BM B cells was associated with an increased rate of apoptosis, and we established that T. cruzi trypomastigotes failed to directly induce immature B-cell apoptosis. We proved that this cell death process occurs in a Fas/FasL-independent fashion but depends on the presence of $\mathrm{CD}_{11 b^{+}}$myeloid cells that secrete a product of the cyclooxygenase pathway that depletes immature B cells [19].
In addition, $\mathrm{BM}$ is compromised in other protozoan parasite infections. In fact, infections with Neospora caninum [20] and T. brucei [21] also cause a general decrease in BM cells. Recently, the T. brucei infection upshot on B lymphopoiesis has been examined using a C57BL/6 mouse T. brucei AnTat 1.1E infection model [22]. Using this model, Bockstal et al. [23] observed that the number of hematopoietic stem cells was minimally affected, but BM B lymphopoiesis was severely affected in $T$. brucei-infected mice, starting with the common lymphoid progenitor fraction. The pre-proB-cell population showed a $50 \%$ reduction by day 20 after infection, while the subsequent B-cell maturation stage, that is, the pro-B, pre-B, and immature $\mathrm{B}$-cell populations reached more than $95 \%$ depletion by day 10 after infection and failed to recover throughout the further course of infection. In T. brucei infection, mice do not present increased apoptosis of BM B-cell precursors nor alteration in the expression of B-cell-development-specific transcription factors like Icaros, PU.1, EBF and E2A and the IL-7. However, T. brucei-infected mice show a reduction in BM CXCL12 levels [23], indicating that during early T. brucei infections, B-cell precursors prematurely migrate out of the $\mathrm{BM}$ as a result of the initiation of inflammation. Similarly, CXCL12 decreased production by BM cells was determined in Plasmodium chabaudi infection [24]. Furthermore, the significant reduction in CXCL12 expression in the BM of 10 days $P$. chaubaudi-infected mice correlates with a reduction in B-cell precursor cells. At days 20 and 30 of infection, a significant recovery in CXCL12 expression in BM is detected, coinciding with a slow recovery of B lymphopoiesis.

\section{B1 and MZ B-Cell Response in Protozoan Parasite Infections}

Among the mature B cells, MZ and B1 B cells appear to be evolutionarily selected and maintained to facilitate prompt $\mathrm{Ab}$ responses. Due to this, they provide a bridge between the innate and the adaptive arms of the antipathogen immune response. B1 cells, distinguished from B2 cells by their phenotype $\left(\mathrm{B} 220^{\text {low }} \mathrm{CD}^{+/-} \mathrm{CD} 1 \mathrm{~b}^{+}\right)$and anatomic location and functional properties, are the dominant population of $B$ cells in the pleural and peritoneal cavities, but represent only a small fraction of splenic B cells [25]. B1 cells produce most of the natural serum IgM and much of the gut IgA and express a BCR repertoire that is enriched for highly polyspecific receptors with low affinities to a broad range of antigens [26]. Despite the fact that B1 cells are very efficient in the control of bacterial and viral infections, they apparently do not play a role in the control of protozoan parasite replication. Indeed, BALB/c Xid mice carrying an X-linked mutation (that prevents B1 cell development) infected with T. cruzi display poor B-cell responses to the infection, accompanied by low levels of specific and nonspecific immunoglobulins in the serum [27]. Surprisingly, Xid mice infected with $T$. cruzi were able to control parasitemia and did not show the wasting syndrome observed in wild-type mice. In addition, they developed almost no pathology early in the chronic phase. The resistance of these mice to experimental Chagas disease was associated with the absence of 


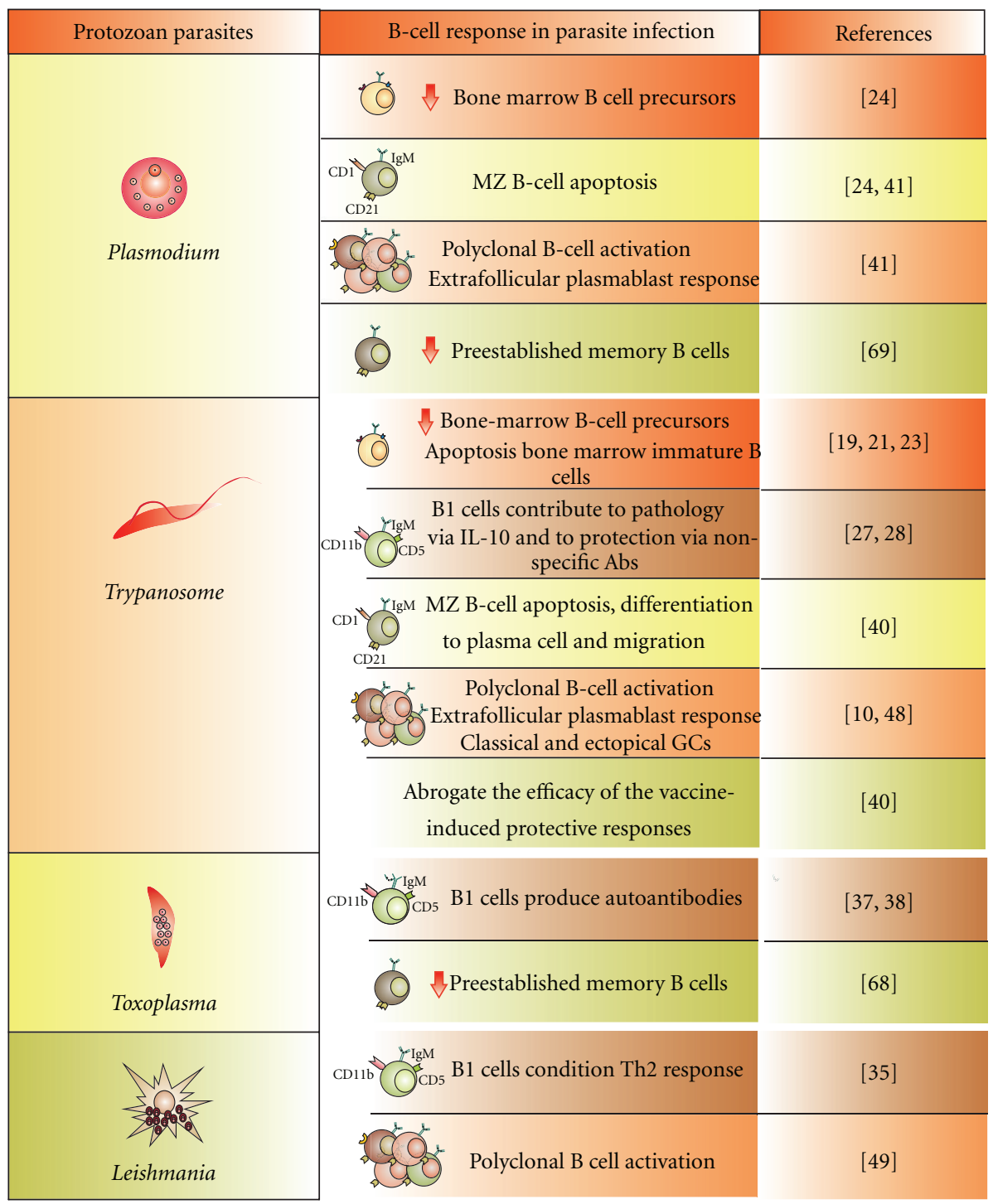

Figure 1: Protozoan parasites affect the different B-cell compartments. MZ: marginal zone B cells, GCs: germinal centers.

IL-10-secreting B1 cells and high levels of IFN-gamma [28]. These results suggested that B1 cells play a pathological rather than protective role in Chagas' disease. Additionally, in T. cruzi infection, we observed a disappearance of peritoneal B1 cells, due to an enhanced differentiation into a particular type of plasma cells, the "Mott-like cells" [29]. Nevertheless, the specific role of these cells in the experimental Chagas disease has not been elucidated yet; their association with autoimmune manifestations in CD22-deficient mice [30] and lupus $[31,32]$ suggests that these cells may be involved in the autoimmune responses observed in T. cruzi infection. We and others have reported that the peritoneal B-cell response observed in T. cruzi infection is almost not specific for the invading pathogen $[29,33]$. However, these "sticky" antibodies could unspecifically bind to parasites providing protection.

B1 cells are not only implicated on Ab secretion; in fact, they may modulate T-cell response. In this sense, O'Garra et al. [34] have reported that B1 cells secrete large amounts of
IL-10 and, consequently, can contribute to the susceptibility of BALB/c mice to L. major infection by skewing the Thelper cell network towards a Th2 phenotype. In this way, it has been observed that $L$. major infection of B-cell-defective $\mathrm{BALB} / \mathrm{c}$ Xid mice induces a less severe disease compared to wild-type control mice [35]. Another report indicates that the behavior of $L$. major-infected Xid mice can be explained more in relation to the high endogenous IFN-gamma production than to the lack of B1 cells. Indeed, B1-cell-depleted irradiated mice showed similar or even worse disease progression compared to control BALB/c mice [36].

B1 cells would also be implicated in the pathogenesis of toxoplasmosis through the production of Abs against the heat shock protein 70 of $T$. gondii that also recognize mice HSP70 [37]. These Abs seem to have a pathogenic role in toxoplasmosis as their injection in $T$. gondii-infected mice clearly increases the number of parasites in mice brain [38]. Moreover, IL-10 produced by B1 cells could, in turn, favor $T$. gondii survival. Then, fine tune regulation of the exacerbated 
Th1 response by IL-10 is important during T. gondii infection.

MZ B cells are also considered innate-like cells that can be induced to differentiate into short-lived plasma cells in the absence of BCR ligation. Splenic MZ B cells can be distinguished from the other splenic B cells by CD24 high, $\mathrm{IgM}^{\text {high }}, \mathrm{IgD}^{\text {high }}, \mathrm{CD}^{2} 3^{-}$expression, as well as by their higher expression of CD21. It is known that these B cells mediate humoral immune responses against blood-borne type $2 \mathrm{~T}$ independent antigens [39] but their role in parasite infection has been scarcely studied. Induction of a T-independent anti-trypanosome IgM response has been shown to be a crucial factor in T. brucei parasite elimination [1]. Even when increased splenic cellularity occurs after T. brucei infection, a significant reduction of splenic IgM+ MZ B-cell numbers takes place right after the first week of infection [40]. The infection-associated disappearance of the MZ B cells from the spleen could be explained by two independent mechanisms, namely, cell differentiation and/or cell death. Supporting the first possibility is the observation that the rapid disappearance of MZ B cells coincided with the temporary accumulation of IgM+ plasma cells. The analysis of MZ B cells that remain in the spleen in the days following the clearance of the first peak of parasitemia revealed that these cells upregulated Annexin V expression. In addition, these cells exhibit caspase 3 gene expression as well as the conversion of procaspase 3 into the cleaved $12 \mathrm{kD}$ and $17 \mathrm{kD}$ caspase 3 activation products suggesting the induction of trypanosomiasis-associated apoptosis in the splenic MZ B-cell population [40]. As in African trypanosomes, P. chabaudi chabaudi infection also caused a severe depletion of MZ B cells in the spleen [41] and this loss is mainly the result of the highly increased rate of apoptosis [24]. As MZ B cells serve as an important source for T-cell independently generated IgM+ plasma cells during early stages of infection, apoptosis induction of MZ B cells can be used by parasites as strategy to avoid early IgM protective response and, consequently, prolong their survival.

\section{Protozoan Infections Induce Massive B-Cell Response with Polyclonal Activation of Splenic B Cells}

Whereas the BM of some protozoan-infected mice suffers from a strong B-lineage-cell depletion, the spleens show a marked cellular hyperplasia as a consequence of an intense $\mathrm{B}$-cell response. A detailed analysis of splenic B-cell response was performed in experimental Chagas disease and malaria $[10,41]$. An extrafollicular Ab response, in mice infected with T. cruzi, is evident a few days after infection and reached a peak after 18 days of infection. This extended kinetics of the extrafollicular response could be characteristic of infections caused by blood circulating protozoan parasites since in $P$. chabaudi chabaudi infection extrafollicular plasmablasts are visible from day 4 , and by day 10 they are unconventionally sited in the periarteriolar region of the white pulp. In this region, in both T. cruzi and Plasmodium infection, extrafollicular plasmablasts form clusters occupying part of the area normally filled by $\mathrm{T}$ cells. The kinetics of the appearance of GCs during T. cruzi and Plasmodium infection are similar to those observed after immunization with classical haptenated proteins, where GCs are visible within the 8 days of immunization [42]. In addition, we detected functional ( $\mathrm{Ab}$ producing) GCs in atypical sites. The GCs in the spleens of $T$. cruzi-infected mice persisted for at least 32 days resembling the kinetics of the response seen in P. chabaudi [41] and $L$. amazonensis [43] infections. We observed that even though T. cruzi infection induces early, persistent, and massive extrafollicular and follicular plasmablast responses together with classical and ectopic GCs, infected mice have a delayed parasite-specific $\mathrm{Ab}$ response. A key finding of our study [10] is that, while an important amount of Abs is rapidly secreted during infection, antigen specific antibodies were not detected until the third week of infection.

The consequence of the massive extrafollicular and follicular B-cell response is the polyclonal B-cell activation that leads to hypergammaglobulinemia with serum Abs specific for the parasite and self- and/or nonrelated Ags [44-46]. In leishmaniasis, hypergammaglobulinemia was described in both $L$. major-susceptible and -resistant mouse strains. T. congolense infection also results in a strong production of non-parasite-specific Abs characterized by the predominance of IgG2a- and IgG2b isotypes [47]. All the mouse strains infected with $T$. congolense present a marked increase in splenic B cells resulting in a nonspecific polyclonal activation of lymphocytes that affects primarily B cells. In strains of $T$. congolense mice which survived longest, that is, $\mathrm{C} 57 \mathrm{~B} 1 / 6 \mathrm{~J}$ and $\mathrm{AKR} / \mathrm{A}$, the increase in splenic $\mathrm{B}$ cells is less marked.

Different roles are proposed for polyclonal B-cell activation, which can be crucial for early host defense by contributing with Abs specific for a spectrum of conserved structures present in the pathogens. Additionally, polyclonal activation can be a mechanism triggered by microorganisms to escape the host-specific immune response by diluting pathogen-specific Abs while increasing irrelevant antibodies. Accordingly, recently it has been reported that $\mathrm{C} 57 \mathrm{Bl} / 6$ mice, resistant to T. cruzi infection, had improved parasite-specific humoral responses that were associated with decreased polyclonal B-cell activation. In the context of parasite infection, Bryan et al. [48] study shows that Th2 cytokine responses were associated with amplified polyclonal B-cell activation and diminished specific humoral immunity. This report demonstrate, that polyclonal B-cell activation during acute experimental Chagas disease is not a generalized response and suggests that the nature of humoral immunity during $T$. cruzi infection contributes to host susceptibility. In leishmaniasis visceral, at early times after infection, there is a marked B-cell expansion in the draining lymph nodes of the site of the infection, which persists throughout infection. As early as day 7 after infection, polyclonal antibodies (TNP, OVA, chromatin) were observed in infected mice and the levels appeared comparable to the specific antileishmania response. Although B-cell-deficient JhD BALB/C mice are relatively resistant to infection, neither B-cell-derived IL-10 nor B-cell antigen presentation appears to be primarily responsible for the elevated parasitemia. Interestingly, passive transfer and 
reconstitution of JhD BALB/c with secretory immunoglobulins (IgM or IgG; specific or nonspecific immune complexes) results in increased susceptibility to $L$. infantum infection [49].

Another potential deleterious role for polyclonal activation is that it could potentially turn on anti-self-responses and lead to autoimmune manifestations during chronic infections. IgG autoantibodies to brain antigens are increased in $P$. falciparum-infected patients and correlate with disease severity in African children [50]. Autoreactive Abs against endocardium and nerves can be detected in mice and humans infected with T. cruzi $[51,52]$ and are thought to be responsible for much of the Chagas' disease pathological damage. Recently, we reported that BAFF-BAFF-R signaling in T. cruzi infection partially controls polyclonal B-cell response but not parasite-specific class-switched primary effectors B cells. BAFF (TNF superfamily B lymphocyte stimulator), a crucial factor for the survival of peripheral $\mathrm{B}$ cells [53-55] associated to the development of autoimmune disorders [56], is produced early and persists throughout the infection with T. cruzi. By BAFF blockade we observed that this cytokine mediates the mature $\mathrm{B}$-cell response and the production of non-parasite-specific IgM and IgG and influences the development of antinuclear IgG [57].

In addition, polyclonal B-cell activation can be responsible for maintenance of memory B-cell responses because of the continuous, unrestricted stimulation of memory B cells whose $\mathrm{Ab}$ production may be sustained in the absence of the antigens binding-specific BCR [58].

\section{B Cells Influence the Characteristic of Cellular Immune Response Because They Act as APC and Cytokine/Chemokine Producers}

Besides being the precursors of the Ab-secreting cells, B cells are committed to do other immune functions such as $\mathrm{Ag}$ presentation to $\mathrm{T}$ cells or cytokine/chemokine production. It has been widely studied that $\mathrm{CD} 8^{+} \mathrm{CTL}$ are important for protective T. cruzi immunity $[59,60]$ but generally they are not induced by soluble protein vaccines. However, a mechanism known as cross-priming has been described whereby certain professional APC can induce $\mathrm{CD} 8^{+} \mathrm{T}$-cell responses after the uptake of exogenous Ag [61]. Hoft et al. [62] have demonstrated that the APC functions of B cells may be important for the induction of optimal vaccineinduced responses in mice immunized with a mix of $\mathrm{CpG}$ and $T$. cruzi transsialidase, an enzyme involved in parasite infectivity. They also demonstrate that mice deficient in B cells (uMT mice) fails to induce protective immunity when they were immunized with $\mathrm{CpG}$ and T. cruzi transsialidase. This failure of uMT mice to be protected was associated with the absence of T. cruzi transsialidase-specific $\mathrm{CD}^{+} \mathrm{T}$ cell response, suggesting that B cells may be important for the cross-priming of $\mathrm{CD} 8^{+} \mathrm{CTL}$. In addition, it has been reported that $T$. cruzi-infected B-cell-deficient mice have reduced numbers of $\mathrm{CD}^{+}$splenic $\mathrm{T}$ cells and impaired generation of central or effector splenic memory $\mathrm{T}$ cells [63]. T. gondii-infected C57BL/6 mice develop a robust and uncontrolled Th1 response, and it has been reported that T. gondii-primed B cells, but not naive B cells, were able to increase IFN gamma production by splenic $\mathrm{T}$ cells in vivo. The mechanisms involved may be linked to the presence of membrane-bound TNF on B-cell surface [64].

The fine tune regulation of migratory cells during infection is an important event in which chemokines and their receptors play a leading role. Different reports have demonstrated that the chemokine receptor CCR5 plays a role in systemic protection and cardiac inflammation during T. cruzi infection $[65,66]$. $\mathrm{CCR}^{+}$cells migrate to both mucosal and systemic sites in response to the chemokines CCL3 (MIP-1a), CCL4 (MIP-1b), and CCL5 (RANTES). In line with these reports, Sullivan et al. [67] showed that neutralization of CCL5 in CCR5 ${ }^{-/-}$T. cruzi-immune mice results in decreased levels of T. cruzi-specific B cell responses and decreased mucosal protection in these mice. They also showed that CCL5 produced by B cells acts in an autocrine manner to increase B cell proliferation and total IgM secretion.

\section{Established Memory B Cell Response Is Affected by Protozoan Parasite Infections}

A hallmark of adaptive immunity is the ability to generate humoral immunological memory by which memory B cells could respond more rapidly and robustly to re-exposure to a new infection. Interestingly, it has been reported that T. brucei infection is capable of abrogating the efficacy of the vaccine-induced protective responses against nonrelated pathogens such as $B$. pertussis [40]. In the same line, Strickland and Sayles [68] showed that T. gondii infected mice which were immunized with SRBC had a depression not only in the primary, but also in the secondary humoral immune response, since they showed less IgM and IgG splenic Ab-secreting cells than non-infected control mice. All the data discussed in the present review indicate that protozoan parasites not only affect the development of the cells involved in Ab production [69] but also affect an already established humoral response against other pathogens. Then, the identification of mechanisms able to improve B cell response and, consequently, parasite control will also be beneficial to avoid the deterioration of a memory response to other pathogens.

\section{Conflict of Interests}

The authors have declared that no competing interests exist.

\section{Acknowledgments}

This work received financial support from CONICET, ANPCyT, and SECYT-UNC to A. Gruppi. We would like to thank to the National Institute of Vaccine (CNPq-Brazil) for paying the charges of this publication.

\section{References}

[1] S. Magez, A. Schwegmann, R. Atkinson et al., "The role of B-cells and IgM antibodies in parasitemia, anemia, and VSG 
switching in Trypanosoma brucei-infected mice," PLoS Pathogens, vol. 4, no. 8, Article ID e1000122, 2008.

[2] T. von der Weid, N. Honarvar, and J. Langhorne, "Genetargeted mice lacking B cells are unable to eliminate a blood stage malaria infection," Journal of Immunology, vol. 156, no. 7, pp. 2510-2516, 1996.

[3] S. Kumar and R. L. Tarleton, "The relative contribution of antibody production and CD8+ $\mathrm{T}$ cell function to immune control of Trypanosoma cruzi," Parasite Immunology, vol. 20, no. 5, pp. 207-216, 1998.

[4] F. E. Lund, "Cytokine-producing B lymphocytes-key regulators of immunity," Current Opinion in Immunology, vol. 20, no. 3, pp. 332-338, 2008.

[5] C. D. Myers, "Role of B cell antigen processing and presentation in the humoral immune response," FASEB Journal, vol. 5, no. 11, pp. 2547-2553, 1991.

[6] G. J. Silverman and D. A. Carson, "Roles of B cells in rheumatoid arthritis," Arthritis Research and Therapy, vol. 5, no. 4, pp. S1-S6, 2003.

[7] P. Youinou, "B cell conducts the lymphocyte orchestra," Journal of Autoimmunity, vol. 28, no. 2-3, pp. 143-151, 2007.

[8] I. A. Abrahamsohn and W. D. Silva, "Antibody dependent cellmediated cytotoxcity against Trypanosoma cruzi," Parasitology, vol. 75, no. 3, pp. 317-323, 1977.

[9] L. F. Umekita, H. A. Takehara, and I. Mota, "Role of the antibody Fc in the immune clearance of Trypanosoma cruzi," Immunology Letters, vol. 17, no. 1, pp. 85-89, 1988.

[10] D. A. Bermejo, M. C. Amezcua Vesely, M. Khan et al., "Trypanosoma cruzi infection induces a massive extrafollicular and follicular splenic B-cell response which is a high source of non-parasite-specific antibodies," Immunology, vol. 132, no. 1, pp. 123-133, 2011.

[11] C. Ronet, H. Voigt, H. Himmelrich et al., "Leishmania majorspecific B cells are necessary for Th2 cell development and susceptibility to L. major LV39 in BALB/c mice," Journal of Immunology, vol. 180, no. 7, pp. 4825-4835, 2008.

[12] R. Carsetti, "The development of B cells in the bone marrow is controlled by the balance between cell-autonomous mechanisms and signals from the microenvironment," Journal of Experimental Medicine, vol. 191, no. 1, pp. 5-8, 2000.

[13] M. Qing, D. Jones, and T. A. Springer, "The chemokine receptor CXCR4 is required for the retention of $\mathrm{B}$ lineage and granulocytic precursors within the bone marrow microenvironment," Immunity, vol. 10, no. 4, pp. 463-471, 1999.

[14] T. Nagasawa, "Microenvironmental niches in the bone marrow required for B-cell development," Nature Reviews Immunology, vol. 6, no. 2, pp. 107-116, 2006.

[15] J. B. Chung, M. Silverman, and J. G. Monroe, "Transitional B cells: step by step towards immune competence," Trends in Immunology, vol. 24, no. 6, pp. 342-348, 2003.

[16] T. T. Su, B. Guo, B. Wei, J. Braun, and D. J. Rawlings, "Signaling in transitional type $2 \mathrm{~B}$ cells is critical for peripheral B-cell development," Immunological Reviews, vol. 197, pp. 161-178, 2004.

[17] L. A. Herzenberg, "B-1 cells: the lineage question revisited," Immunological Reviews, vol. 175, pp. 9-22, 2000.

[18] E. Montecino-Rodriguez, H. Leathers, and K. Dorshkind, "Identification of a B-1 B cell-specified progenitor," Nature Immunology, vol. 7, no. 3, pp. 293-301, 2006.

[19] E. Zuniga, E. Acosta-Rodriguez, M. C. Merino, C. Montes, and A. Gruppi, "Depletion of immature B cells during Trypanosoma cruzi infection: involvement of myeloid cells and the cyclooxygenase pathway," European Journal of Immunology, vol. 35, no. 6, pp. 1849-1858, 2005.
[20] L. Teixeira, A. Marques, C. S. Meireles et al., "Characterization of the B-cell immune response elicited in BALB/c mice challenged with Neospora caninum tachyzoites," Immunology, vol. 116, no. 1, pp. 38-52, 2005.

[21] C. E. Clayton, M. E. Selkirk, and C. A. Corsini, "Murine trypanosomiasis: cellular proliferation and functional depletion in the blood, peritoneum, and spleen related to changes in bone marrow stem cells," Infection and Immunity, vol. 28, no. 3, pp. 824-831, 1980.

[22] N. Van Meirvenne, E. Magnus, and P. Büscher, "Evaluation of variant specific trypanolysis tests for serodiagnosis of human infections with Trypanosoma brucei gambiense," Acta Tropica, vol. 60, no. 3, pp. 189-199, 1996.

[23] V. Bockstal, P. Guirnalda, G. Caljon et al., "T. brucei infection reduces $\mathrm{B}$ lymphopoiesis in bone marrow and truncates compensatory splenic lymphopoiesis through transitional Bcell apoptosis," PLoS Pathogens, vol. 7, no. 6, Article ID e1002089, 2011.

[24] V. Bockstal, N. Geurts, and S. Magez, "Acute disruption of bone marrow B lymphopoiesis and apoptosis of transitional and marginal zone B cells in the spleen following a blood-stage plasmodium chabaudi infection in mice," Journal of Parasitology Research, vol. 2011, Article ID 534697, 11 pages, 2011.

[25] K. Hayakawa and R. R. Hardy, "Development and function of B-1 cells," Current Opinion in Immunology, vol. 12, no. 3, pp. 346-354, 2000.

[26] N. Baumgarth, J. W. Tung, and L. A. Herzenberg, "Inherent specificities in natural antibodies: a key to immune defense against pathogen invasion," Springer Seminars in Immunopathology, vol. 26, no. 4, pp. 347-362, 2005.

[27] P. Minoprio, A. Coutinho, S. Spinella, and M. HontebeyrieJoskowicz, "Xid immunodeficiency imparts increased parasite clearance and resistance to pathology in experimental Chagas' disease," International Immunology, vol. 3, no. 5, pp. 427-433, 1991.

[28] P. Minoprio, M. C. El Cheikh, E. Murphy et al., "Xid-associated resistance to experimental Chagas' disease is IFN- $\gamma$ dependent," Journal of Immunology, vol. 151, no. 8, pp. 42004208, 1993.

[29] M. C. Merino, C. L. Montes, E. V. Acosta-Rodriguez, D. A. Bermejo, M. C. Amezcua-Vesely, and A. Gruppi, "Peritoneum from Trypanosoma cruzi-infected mice is a homing site of Syndecan-1neg plasma cells which mainly provide non-parasite-specific antibodies," International Immunology, vol. 22, no. 5, pp. 399-410, 2010.

[30] J. Jellusova, U. Wellmann, K. Amann, T. H. Winkler, and L. Nitschke, "CD22 x Siglec-G double-deficient mice have massively increased B1 cell numbers and develop systemic autoimmunity," Journal of Immunology, vol. 184, no. 7, pp. 3618-3627, 2010.

[31] Z. Xu, E. J. Butfiloski, E. S. Sobel, and L. Morel, “Mechanisms of peritoneal B-1a cells accumulation induced by murine lupus susceptibility locus Sle2," Journal of Immunology, vol. 173, no. 10, pp. 6050-6058, 2004.

[32] T. Ito, S. Ishikawa, T. Sato et al., "Defective B1 cell homing to the peritoneal cavity and preferential recruitment of B1 cells in the target organs in a murine model for systemic lupus erythematosus," Journal of Immunology, vol. 172, no. 6, pp. 3628-3634, 2004.

[33] B. Reina-San-Martin, A. Cosson, and P. Minoprio, "Lymphocyte polyclonal activation: a pitfall for vaccine design against infectious agents," Parasitology Today, vol. 16, no. 2, pp. 6267, 2000. 
[34] A. O’Garra, R. Chang, N. Go, R. Hastings, G. Haughton, and M. Howard, "Ly-1 B (B-1) cells are the main source of B cellderived interleukin 10," European Journal of Immunology, vol. 22, no. 3, pp. 711-717, 1992.

[35] A. Hoerauf, W. Solbach, M. Lohoff, and M. Rollinghoff, "The Xid defect determines an improved clinical course of murine leishmaniasis in susceptible mice," International Immunology, vol. 6, no. 8, pp. 1117-1124, 1994.

[36] B. Babai, H. Louzir, P. A. Cazenave, and K. Dellagi, "Depletion of peritoneal CD5+ B cells has no effect on the course of Leishmania major infection in susceptible and resistant mice," Clinical and Experimental Immunology, vol. 117, no. 1, pp. 123129, 1999.

[37] M. Chen, F. Aosai, H. S. Mun, K. Norose, H. Hata, and A. Yano, "Anti-HSP70 autoantibody formation by B-1 cells in Toxoplasma gondii-infected mice," Infection and Immunity, vol. 68, no. 9, pp. 4893-4899, 2000.

[38] M. Chen, F. Aosai, K. Norose, H. S. Mun, and A. Yano, "The role of anti-HSP70 autoantibody-forming VH1-JH1 B-1 cells in Toxoplasma gondii-infected mice," International Immunology, vol. 15, no. 1, pp. 39-47, 2003.

[39] F. Martin, A. M. Oliver, and J. F. Kearney, "Marginal zone and $\mathrm{B} 1 \mathrm{~B}$ cells unite in the early response against T-independent blood-borne particulate antigens," Immunity, vol. 14, no. 5, pp. 617-629, 2001.

[40] M. Radwanska, P. Guirnalda, C. De Trez, B. Ryffel, S. Black, and S. Magez, "Trypanosomiasis-induced B cell apoptosis results in loss of protective anti-parasite antibody responses and abolishment of vaccine-induced memory responses," PLoS Pathogens, vol. 4, no. 5, Article ID e1000078, 2008.

[41] A. H. Achtman, M. Khan, I. C. M. MacLennan, and J. Langhorne, "Plasmodium chabaudi chabaudi infection in mice induces strong B cell responses and striking but temporary changes in splenic cell distribution," Journal of Immunology, vol. 171, no. 1, pp. 317-324, 2003.

[42] J. Jacob, R. Kassir, and G. Kelsoe, "In situ studies of the primary immune response to (4-hydroxy-3-nitrophenyl)acetyl. I. The architecture and dynamics of responding cell populations," Journal of Experimental Medicine, vol. 173, no. 5, pp. 1165-1175, 1991.

[43] A. L. Abreu-Silva, K. S. Calabrese, S. M. N. Cupolilo, F. O. Cardoso, C. S. F. Souza, and S. C. Gonçalves Da Costa, "Histopathological studies of visceralized Leishmania (Leishmania) amazonensis in mice experimentally infected," Veterinary Parasitology, vol. 121, no. 3-4, pp. 179-187, 2004.

[44] C. Daniel-Ribeiro, J. de Oliveira-Ferreira, D. M. Banic, and B. Galvao-Castro, "Can malaria-associated polyclonal B-lymphocyte activation interfere with the development of antisporozoite specific immunity?" Transactions of the Royal Society of Tropical Medicine and Hygiene, vol. 83, no. 3, pp. 289-292, 1989.

[45] R. E. Sacco, M. Hagen, J. E. Donelson, and R. G. Lynch, "B lymphocytes of mice display an aberrant activation phenotype and are cell cycle arrested in G0/G(1A) during acute infection with Trypanosoma brucei," Journal of Immunology, vol. 153, no. 4, pp. 1714-1723, 1994.

[46] P. Minoprio, "Parasite polyclonal activators: new targets for vaccination approaches?" International Journal for Parasitology, vol. 31, no. 5-6, pp. 588-591, 2001.

[47] W. I. Morrison, G. E. Roelants, K. S. Mayor-Withey, and M. Murray, "Susceptibility of inbred strains of mice to Trypanosoma congolense: correlation with changes in spleen lymphocyte populations," Clinical and Experimental Immunology, vol. 32, no. 1, pp. 25-40, 1978.
[48] M. A. Bryan, S. E. Guyach, and K. A. Norris, "Specific humoral immunity versus polyclonal B Cell activation in trypanosoma cruzi infection of susceptible and resistant mice," PLoS Neglected Tropical Diseases, vol. 4, no. 7, article e733, 2010.

[49] E. Deak, A. Jayakumar, K. W. Cho et al., "Murine visceral leishmaniasis: IgM and polyclonal B-cell activation lead to disease exacerbation," European Journal of Immunology, vol. 40, no. 5, pp. 1355-1368, 2010.

[50] V. Guiyedi, Y. Chanseaud, C. Fesel et al., "Self-reactivities to the non-erythroid alpha spectrin correlate with cerebral malaria in gabonese children," PLoS One, vol. 2, no. 4, article e389, 2007.

[51] J. M. Peralta, P. Ginefra, J. C. Dias, J. M. Magalhaes, and A. Szarfman, "Autoantibodies and chronic Chagas's heart disease," Transactions of the Royal Society of Tropical Medicine and Hygiene, vol. 75, no. 4, pp. 568-569, 1981.

[52] A. Szarfman, A. Luquetti, and A. Rassi, "Tissue-reacting immunoglobulins in patients with different clinical forms of Chagas' disease," The American Journal of Tropical Medicine and Hygiene, vol. 30, no. 1, pp. 43-46, 1981.

[53] P. A. Moore, O. Belvedere, A. Orr et al., "BLyS: member of the tumor necrosis factor family and B lymphocyte stimulator," Science, vol. 285, no. 5425, pp. 260-263, 1999.

[54] P. Schneider, F. Mackay, V. Steiner et al., "BAFF, a novel ligand of the tumor necrosis factor family, stimulates B cell growth," Journal of Experimental Medicine, vol. 189, no. 11, pp. 17471756, 1999.

[55] M. Batten, J. Groom, T. G. Cachero et al., "BAFF mediates survival of peripheral immature B lymphocytes," Journal of Experimental Medicine, vol. 192, no. 10, pp. 1453-1466, 2000.

[56] F. MacKay and P. Schneider, "Cracking the BAFF code," Nature Reviews Immunology, vol. 9, no. 7, pp. 491-502, 2009.

[57] D. A. Bermejo, M. C. Amezcua-Vesely, C. L. Montes et al., "BAFF mediates splenic B cell response and antibody production in experimental chagas disease," PLoS Neglected Tropical Diseases, vol. 4, no. 5, article e679, 2010.

[58] N. L. Bernasconi, E. Traggiai, and A. Lanzavecchia, "Maintenance of serological memory by polyclonal activation of human memory B cells," Science, vol. 298, no. 5601, pp. 21992202, 2002.

[59] R. L. Tarleton, "Depletion of CD8+ T cells increases susceptibility and reverses vaccine-induced immunity in mice infected with Trypanosoma cruzi," Journal of Immunology, vol. 144, no. 2, pp. 717-724, 1990.

[60] R. L. Tarleton, B. H. Koller, A. Latour, and M. Postan, “Susceptibility of $\beta 2$-microglobulin-deficient mice to Trypanosoma cruzi infection,” Nature, vol. 356, no. 6367, pp. 338-340, 1992.

[61] C. Kurts, B. W. Robinson, and P. A. Knolle, "Cross-priming in health and disease," Nature Reviews Immunology, vol. 10, no. 6, pp. 403-414, 2010.

[62] D. F. Hoft, C. S. Eickhoff, O. K. Giddings, J. R. C. Vasconcelos, and M. M. Rodrigues, "Trans-sialidase recombinant protein mixed with CpG motif-containing oligodeoxynucleotide induces protective mucosal and systemic trypanosoma cruzi immunity involving CD8+ CTL and B cell-mediated crosspriming," Journal of Immunology, vol. 179, no. 10, pp. 68896900, 2007.

[63] F. Cardillo, E. Postol, J. Nihei, L. S. Aroeira, A. Nomizo, and J. Mengel, "B cells modulate $\mathrm{T}$ cells so as to favour T helper type 1 and $\mathrm{CD} 8+\mathrm{T}$-cell responses in the acute phase of Trypanosoma cruzi infection,” Immunology, vol. 122, no. 4, pp. 584-595, 2007.

[64] L. C. Menard, L. A. Minns, S. Darche et al., "B cells amplify IFN- $\gamma$ production by T cells via a TNF- $\alpha$-mediated 
mechanism," Journal of Immunology, vol. 179, no. 7, pp. 48574866, 2007.

[65] J. L. Hardison, R. A. Wrightsman, P. M. Carpenter, W. A. Kuziel, T. E. Lane, and J. E. Manning, "The CC chemokine receptor 5 is important in control of parasite replication and acute cardiac inflammation following infection with Trypanosoma cruzi," Infection and Immunity, vol. 74, no. 1, pp. 135143,2006

[66] F. S. Machado, N. S. Koyama, V. Carregaro et al., "CCR5 plays a critical role in the development of myocarditis and host protection in mice infected with Trypanosoma cruzi," Journal of Infectious Diseases, vol. 191, no. 4, pp. 627-636, 2005.

[67] N. L. Sullivan, C. S. Eickhoff, X. Zhang, O. K. Giddings, T. E. Lane, and D. F. Hoft, "Importance of the CCR5-CCL5 axis for mucosal Trypanosoma cruzi protection and B cell activation," Journal of Immunology, vol. 187, no. 3, pp. 1358-1368, 2011.

[68] G. T. Strickland and P. C. Sayles, "Depressed antibody responses to a thymus dependent antigen in toxoplasmosis," Infection and Immunity, vol. 15, no. 1, pp. 184-190, 1977.

[69] G. E. Weiss, P. D. Crompton, S. Li et al., "Atypical memory B cells are greatly expanded in individuals living in a malariaendemic area," Journal of Immunology, vol. 183, no. 3, pp. 2176-2182, 2009. 

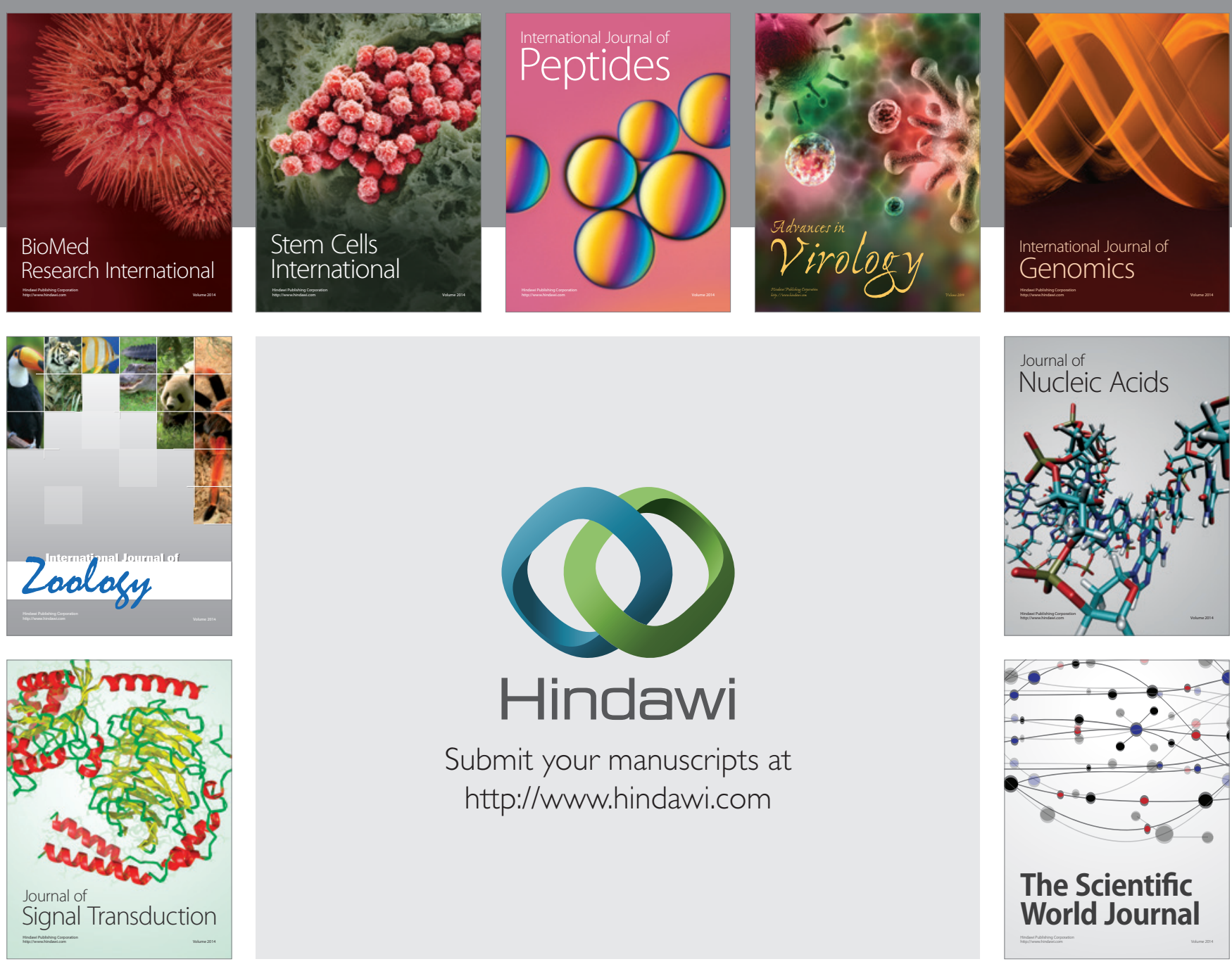

Submit your manuscripts at

http://www.hindawi.com
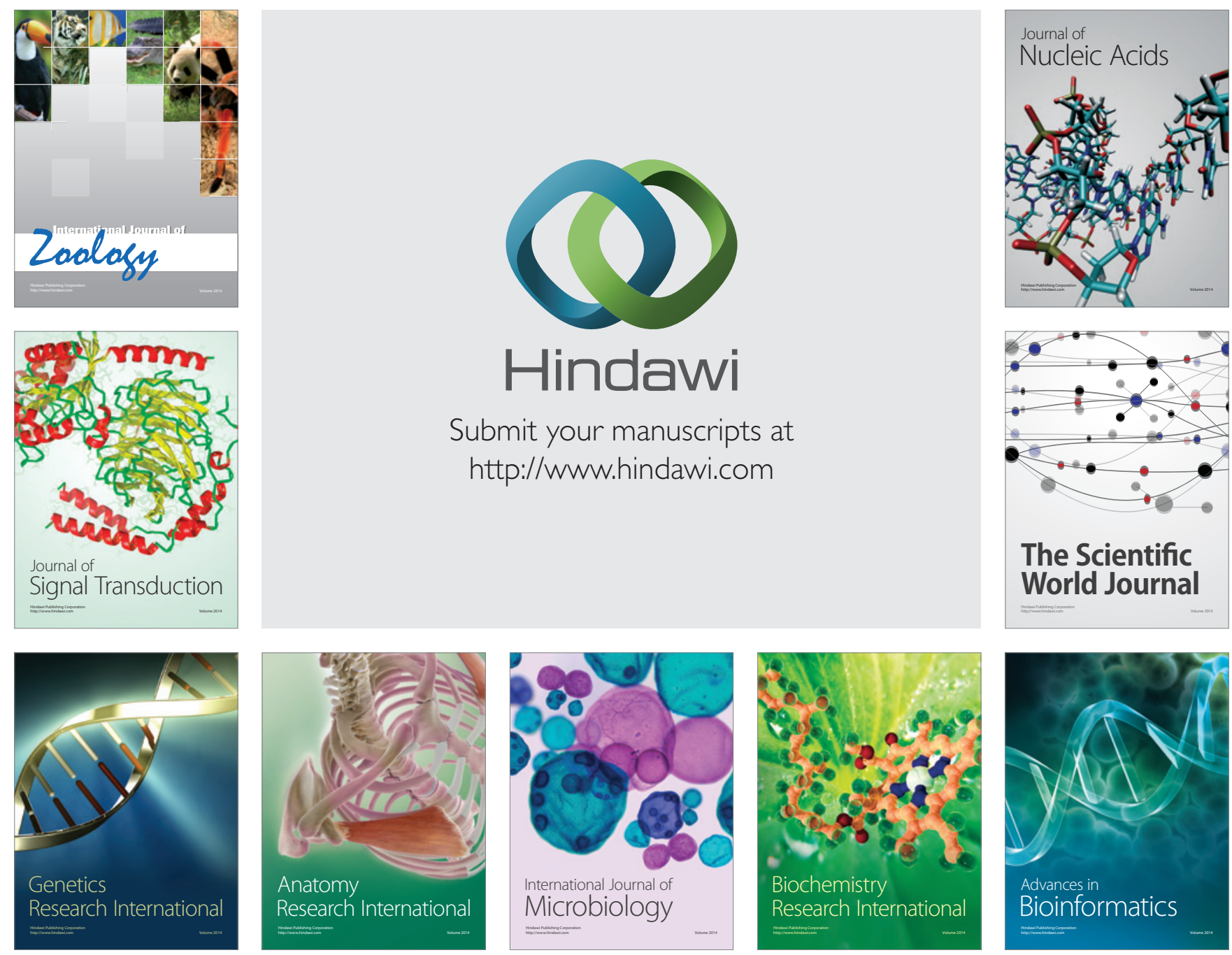

The Scientific World Journal
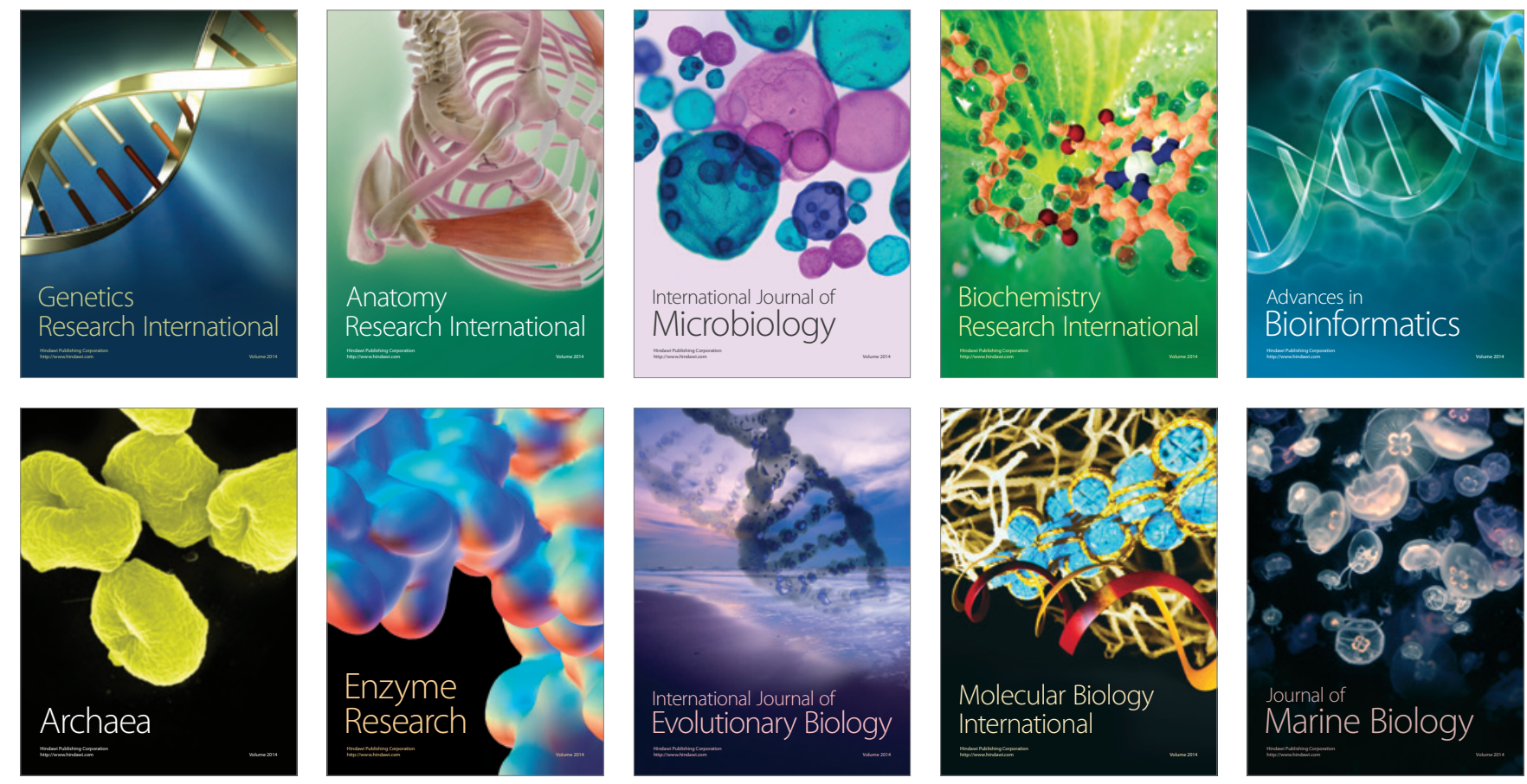